\title{
Cor-triatriatum dexter with pulmonary hypertension in a neonate
}

\author{
Bijay Kumar Meher ${ }^{1 *}$, Pradeep $S^{1}$, Leena Das ${ }^{1}$, Priyadarsi Tripathy ${ }^{2}$ \\ ${ }^{1}$ PG Department of Paediatrics SVPPGIP, SCB Medical College, Cuttack, India \\ ${ }^{2}$ Department of Cardiology, SCB Medical College, Cuttack, India \\ Email: "bkmeher187@yahoo.co.in
}

Received 3 October 2013; revised 30 October 2013; accepted 7 November 2013

Copyright (C 2013 Bijay Kumar Meher et al. This is an open access article distributed under the Creative Commons Attribution License, which permits unrestricted use, distribution, and reproduction in any medium, provided the original work is properly cited.

\begin{abstract}
Cor-triatriatum dexter is an extremely rare congenital anomaly, in which right atrium is divided into 2 halves by a septum giving rise to 3 atrial chambers. We report here a case of cor-triatriatum dexter who presented at 3 days of life with tachypnea and did not respond to antibiotics. Echocardiography revealed ostium secundum atrial septal defect (ASD) with right to left flow, intact interventricular septum (IVS), severe tricuspid regurgitation with pulmonary arterial hypertension and a membranous structure dividing right atrium into 2 halves with good biventricular function. The patient improved with sildenafil and anti-failure medication. This case of cor-triatriatum dexter presenting with pulmonary hypertension, tricuspid regurgitation and ASD added to the new spectrum of cor-triatriatum dexter.
\end{abstract}

Keywords: Cor-Triatriatum; Pulmonary Hypertension; Neonate

\section{INTRODUCTION}

Cor-triatriatum dexter is an extremely rare congenital anomaly [1], in which right atrium is divided into 2 halves by a septum giving rise to 3 atrial chambers. Similarly, cor-triatriatum sinistrum, in which left atrium is divided into 2 halves. Cor-triatriatum incidence is approximately less than $0.1 \%$ of all congenital heart anomalies [2]. Usually, isolated defect is an incidental finding in adults, which does not cause significant disturbances. We had a case of cor-triatriatum dexter which is being described in the following part.

\section{CASE REPORT}

A second order term female baby was born out of non-

"Corresponding author. consanguineous marriage to a 23-year-old mother by normal vaginal delivery, birth weight being 2700 gm \& was normal up to day 3 of life. On day 3 of life baby started to have hurried respirations and treated as a case of bacterial pneumonia but as the baby did not responded to antibiotics referred to us for further management. Mother stated having additional complaints of not feeding well and refusal to feed since 2 days. There was no history of fever or bluish discoloration as described by the mother.

On examination, baby was tachypenic, tachycardiac and in respiratory distress (use of accessory muscle present, retractions), capillary refill time, activity, cry, reflex, tone was normal. There was mild pallor, cyanosis of extremities. There was reduced mobility of skull bones and small size of anterior fontanelle. No other dysmorphism found on clinical examination. Examination of chest revealed bilateral equal air entry and vesicular breath sounds, 1st heart sounds was normally audible, P2 being loud and a pan systolic murmur was audible all over the precordium on auscultation. There was hepatomegaly of $4 \mathrm{~cm}$ along the mid-clavicular line and spleen was not palpable.

Patient was admitted in ICU, sepsis work up was normal. Chest X-ray showed cardiomegaly (C:T ratio $>0.6$ ) with perihilar opacity. ECG showed RVH \& RAH. Bedside ECHO was done, showed congenital acyanotic heart disease, ostium secundum ASD of size $12 \mathrm{~mm}$ with right to left flow, IVS intact, severe tricuspid regurgitation with pulmonary arterial hypertension (30 - $35 \mathrm{mmHg}$ ), a membranous structure dividing right atrium into 2 halves with good biventricular function (Figures 1 and 2) and doppler showed flow across the membrane (Figure 3). The patient was treated with sildenafil for pulmonary hypertension and heart failure medications, the respiratory distress reduced and patient improved symptomatically. Then the patient was shifted to cardiology department for the confirmation of the ECHO findings. The 2nd ECHO findings showed similar findings. The patient 


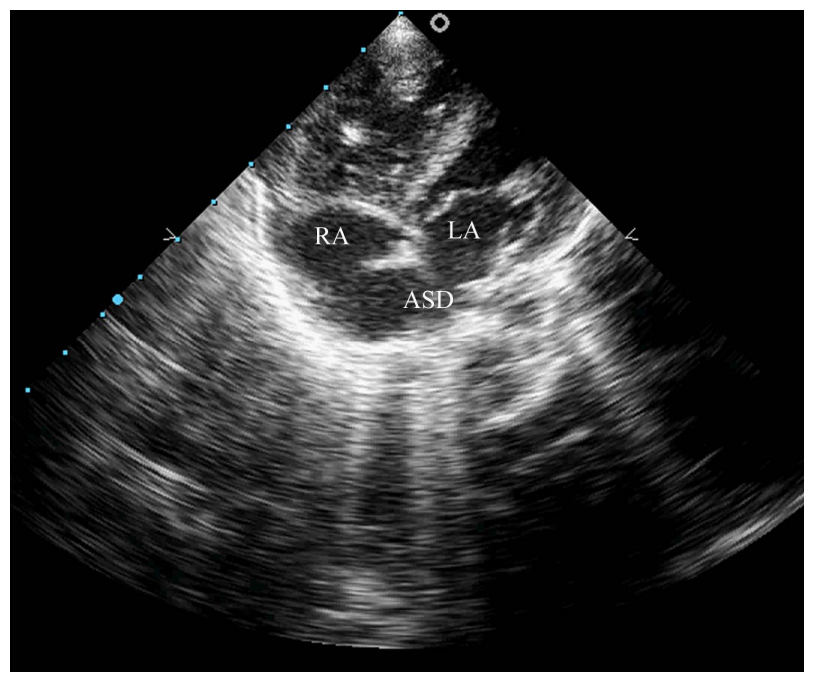

Figure 1. Echo image showing 3 chambers, a separate membrane dividing right atrium into 2 chambers upper and lower chamber.

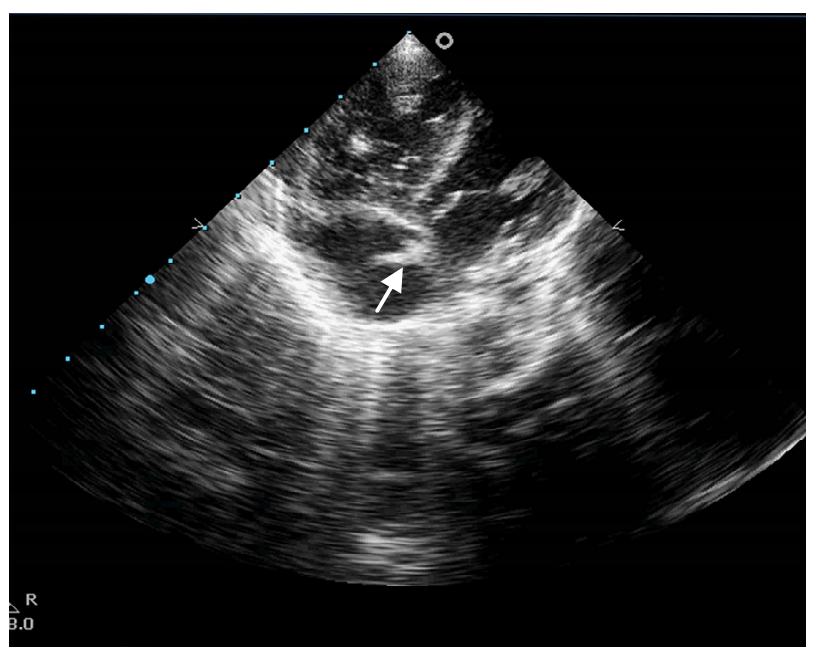

Figure 2. Arrow showing the membrane in right atrium.

was discharged with sildenafil, anti-failure medications and advised for follow-up.

\section{DISCUSSION}

The membrane divides the right atrium into a proximal (upper) and a distal (lower) chamber. The upper chamber receives the venous blood from both vena cava and the lower chamber is in contact with the tricuspid valve and the right atrial appendage [3]. Feto-pathophysiology of cor triatriatum dexter is being complete persistence of right sinus valve of embryonic life causing the division of right atrium during embryogenesis. The right horn of the sinus venosus and the embryologic right atrium are then connected through the Sino atrial orifice, which has on either side the 2 valvular folds called the right and left venous valves of which right direct the blood from right

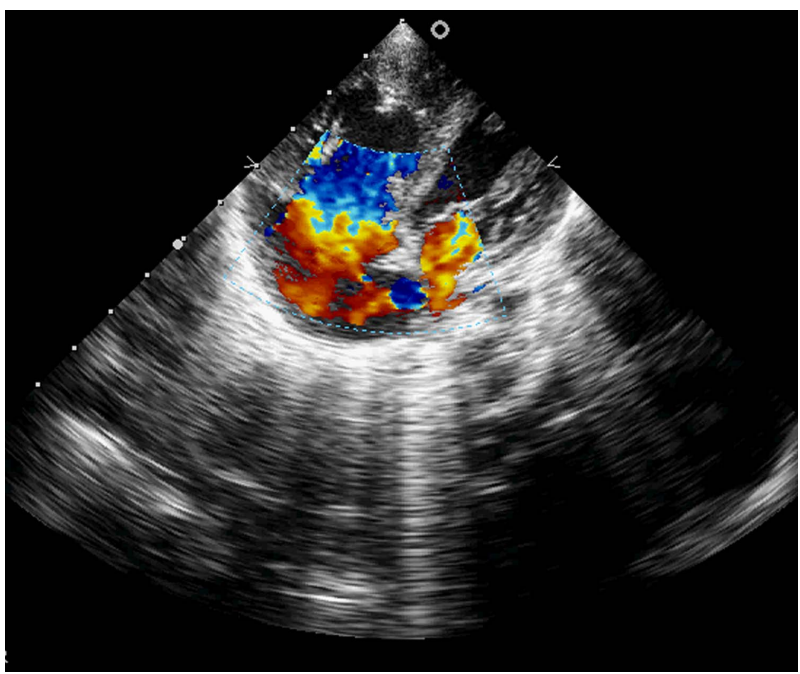

Figure 3. Flow across the membrane is shown, there in flow across ASD and regurgitant flow through tricuspid valve is also shown.

to left atrium. It will normally regress during the development and enlargement of right atrium. If the regression fails that leads to a septum like structure dividing the right atrium into 2 chambers [1,4,5]. When the septation is mild, the condition is often asymptomatic and is an incidental finding during surgery to correct other cardiac abnormalities or during echocardiography. More severe septation can cause right-sided heart failure and elevated central venous pressures due to obstruction of the tricuspid valve, the right ventricular outflow tract, or the inferior vena cava [2]. Clinical presentation is based on Sino IVC-atrial orifice and degree of septation, may have heart murmur, palpitations, raised JVP (jugular venous pressure), supraventricular arrhythmias, abdominal distension, abdominal (hepatic) tenderness and edema [4]. Our case presented with murmur, tachycardia and heaptomegaly. Pulmonary hypertension may be primary as secondary will take long time to develop and our baby was a neonate. Previous reports of Cor triatriatum dextrum usually didn't show association with any lifethreatening symptoms or major congenital cardiac defects. ECG (electrocardiography) no specific features, particularly in isolated case. Our case ECG shows RVH, $\mathrm{RAH}$ and features of pulmonary hypertension. Right atrial catheterization showing difference in pressure were diagnostic in early days (pre-ECHO era), ECHO (both trans thoracic and trans esophageal), cardiac MRI, right ventriculography can show 3 chambers with flow is suggestive of cor-triatrium [6]. Cor-triatriatum can be diagnosed at any age, usually incidentally found during ECHO. Treatment is complete resection of the membrane and closure of the atrial septum with a pericardial patch is a common approach, associated anomalies have also to be corrected [7]. Long-term prognosis for an iso- 
lated cor-triatriatum dexter is good, but there are no reports available for cor-triatriatum dexter associated with pulmonary hypertension. In South India, a case of a divided right atrium evaluated for nonspecific symptoms and unexplained cardiomegaly with right atrial enlargement was reported [8]. Another one case of isolated cor triatriatum dexter, symptomatic for severe central cyanosis, in which the membrane was identified before surgery by means of transthoracic echocardiography alone, and successfully removed surgically at 1 month of life [9].

\section{CONCLUSION}

This is a case report of cor-triatriatum dexter presenting with pulmonary hypertension, tricuspid regurgitation and ASD, which adds to the spectrum of cor-triatriatum dexter. The association and the presentation in neonatal period have not been reported previously.

\section{REFERENCES}

[1] Alboliras, E.T., Edwards, W.D., Driscoll, D.J. and Seward, J.B. (1987) Cor triatriatum dexter: Two-dimensional echocardiographic diagnosis. Journal of the American College of Cardiology, 9, 334-337. http://dx.doi.org/10.1016/S0735-1097(87)80385-6

[2] Ahmad, R., Mohd, Z., Koh, G.T. and Sharifah, A. (2012) Cor triatriatum dexter: A rare cause of childhood cyanosis. Annals of Pediatric Cardiology, 5, 92-94. http://dx.doi.org/10.4103/0974-2069.93725
[3] Hansing, C.E., Young, W.P. and Rowe, G.G. (1972) Cor triatriatum dexter. Persistent right sinus venosus valve. American Journal of Cardiology, 30, 559-564. http://dx.doi.org/10.1016/0002-9149(72)90050-1

[4] Burton, D.A., Chin, A., Weinberg, P.M. and Pigott, J.D. (1987) Identification of Cor triatriatum dexter by twodimensional echocardiography. American Journal of Cardiology, 60, 409-410. http://dx.doi.org/10.1016/0002-9149(87)90268-2

[5] Savas, V., Samyn, J., Schreiber, T.L., Hauser, A. and O’Neill, W.W. (1991) Cor triatriatum dexter: Recognition and percutaneous Trans luminal correction. Catheterization and Cardiovascular Diagnosis, 23, 183-186. http://dx.doi.org/10.1002/ccd.1810230308

[6] Lengyel, M., Arvay, A. and Biro, V. (1987) Two-dimensional echocardiographic diagnosis of Cor triatriatum. American Journal of Cardiology, 59, 484-485. http://dx.doi.org/10.1016/0002-9149(87)90964-7

[7] Ott, D.A., Cooley, D.A., Angelini, P. and Leachman, R.D. (1979) Successful surgical correction of symptomatic Cor triatriatum dexter. Journal of Thoracic and Cardiovascular Surgery, 78, 573-575.

[8] Choudhary, D., Sivasankaran, S., Venkateshwaran, S. and Sasidharan, B. (2013) Cor triatiratum dexter: A rare cause of isolated right atrial enlargement. Pediatric Cardiology, 34, 198-199. http://dx.doi.org/10.1007/s00246-012-0443-0

[9] Galli, M.A., Galletti, L., Schena, F., Salvini, L., Mosca, F. and Danzi, G.B. (2009) A rare case of neonatal cyanosis due "cor triatriatum dexter" and a review of the literature. Journal of Cardiovascular Medicin (Hagerstown), 10, 535538. http://dx.doi.org/10.2459/JCM.0b013e32832a574f 